\title{
CONVERGENCE OF CONDITIONAL EXPECTATIONS AND STRONG LAWS OF LARGE NUMBERS FOR MULTIVALUED RANDOM VARIABLES
}

BY

FUMIO HIAI

\section{Dedicated to Professor Hisaharu Umegaki on his 60 th Birthday}

\begin{abstract}
Fatou's lemmas and Lebesgue's convergence theorems are established for multivalued conditional expectations of random variables having values in the closed subsets of a separable Banach space. Strong laws of large numbers are also given for such multivalued random variables.
\end{abstract}

Introduction. Two notions of convergence for closed subsets in a metric space, the Hausdorff distance convergence and the Kuratowski convergence, are eminently useful in several areas of mathematics and applications like optimization and control, stochastic and integral geometry, mathematical economics, etc. In an (infinite-dimensional) normed space, however, the Mosco convergence [22, 23] is known to be more tractable than the Kuratowski one.

The convergence theorems for multivalued integrals were discussed by Aumann [5], Schmeidler [29], Hildenbrand and Mertens [17], and Artstein [1]. These authors obtained Fatou's lemma and Lebesgue's convergence theorem with the Kuratowski convergence for measurable multivalued functions having values in the closed subsets of $\mathbf{R}^{d}$. Fatou's lemma is of some use in mathematical economics (cf. [17, 29]).

A multivalued strong law of large numbers was first proved by Artstein and Vitale [4] for independent, identically distributed (i.i.d.) random variables whose values are compact subsets of $\mathbf{R}^{d}$. This strong law with the Hausdorff distance convergence has been extended by several authors (see Cressie [8], Hess [12], Puri and Ralescu [25], and Giné, Hahn and Zinn [10]) and was recently completed in [2, 15]. On the other hand, Artstein and Hart [3] obtained a strong law of large numbers with the Kuratowski convergence for i.i.d. random variables having values in the closed subsets of $\mathbf{R}^{d}$, and applied it to a problem of optimal allocations.

This paper has two subjects on the convergence theory for multivalued random variables whose values are closed subsets of a separable Banach space. The first part (§2) gives several results on passage to the limit under the integration of multivalued random variables. The second part (\$3) is concerned with strong laws of large

Received by the editors October 25, 1984.

1980 Mathematics Subject Classification. Primary 60D05, 28B20; Secondary 60F15, 60B12.

Key words and phrases. Multivalued random variable, Mosco convergence, conditional expectation, Fatou's lemma, Lebesgue's convergence theorem, strong law of large numbers. 
numbers for multivalued random variables. Though the two parts are rather disconnected in contents, we discuss them together in this paper because they relate the same type of convergence (the Mosco convergence) and have therefore the same preliminaries.

In $§ 1$, we give definitions and preliminaries on multivalued random variables and the Mosco convergence. In $\$ 2$, we establish Fatou's lemmas and Lebesgue's convergence theorems for multivalued conditional expectations containing multivalued integrals. In $\S 3$, we obtain two types of multivalued strong laws of large numbers with the Mosco convergence. The first is the strong law for i.i.d. multivalued random variables, and the second is for only independent multivalued random variables with some additional conditions. Our main tool in $\$ 3$ is the existence of identically distributed (resp. independent) selections of identically distributed (resp. independent) multivalued random variables, which was presented by Hess [13, 14].

The author wishes to express his gratitude to Professor H. Umegaki for advice and encouragement. He thanks Professor M. Valadier and the referee for useful comments to the original version of this paper.

1. Preliminaries. Throughout this paper, let $(\Omega, \mathscr{A}, \mu)$ be a probability measure space and $\mathfrak{X}$ a real separable Banach space with the dual space $\mathfrak{X}^{*}$. For each $X \subset \mathfrak{X}$, cl $X$, w-cl $X$, and co $X$ denote the norm-closure, the weak-closure, and the closed convex hull of $X$, respectively. Let $\mathscr{K}(\mathfrak{X})$ (resp. $\mathscr{K}_{c}(\mathfrak{X})$ ) denote the family of all nonempty closed (resp. nonempty closed convex) subsets of $\mathfrak{X}$. For $X, Y \in \mathscr{K}(\mathfrak{X})$, the distance $d(y, X)$ of $X$ and $y \in \mathfrak{X}$, the Hausdorff distance $h(X, Y)$ of $X$ and $Y$, the norm $\|X\|$ of $X$, and the support function $s(X, \cdot)$ of $X$ are defined by

$$
\begin{gathered}
d(y, X)=\inf _{x \in X}\|y-x\|, \\
h(X, Y)=\max \left\{\sup _{x \in X} d(x, Y), \sup _{y \in Y} d(y, X)\right\}, \\
\|X\|=h(X,\{0\})=\sup _{x \in X}\|x\|, \\
s\left(X, x^{*}\right)=\sup _{x \in X}\left\langle x, x^{*}\right\rangle, \quad x^{*} \in \mathfrak{X}^{*} .
\end{gathered}
$$

Let $\mathscr{B}_{\mathfrak{X}}$ be the Borel $\sigma$-field on $\mathfrak{X}$ and $\mathscr{B}_{\mathscr{K}(\mathfrak{X})}$ the $\sigma$-field on $\mathscr{K}(\mathfrak{X})$ generated by the sets $\{X \in \mathscr{K}(\mathfrak{X}): X \cap O \neq \varnothing\}$ taken for all open subsets $O$ of $\mathfrak{X}$. A multivalued (set-valued) function $F: \Omega \rightarrow \mathscr{K}(\mathfrak{X})$ is said to be measurable if $F$ is $\left(\mathscr{A}, \mathscr{B}_{\mathscr{K}(\mathfrak{X})}\right)$-measurable, i.e., $F^{-}(O)=\{\omega \in \Omega: F(\omega) \cap O \neq \varnothing\} \in \mathscr{A}$ for every open $O \subset \mathfrak{X}$. Such a function $F$ is called a multivalued (closed-valued) random variable or random closed set. An $F: \Omega \rightarrow \mathscr{K}(\mathfrak{X})$ is measurable if and only if there exists a sequence $\left\{f_{n}\right\}$ of measurable functions $f_{n}: \Omega \rightarrow \mathfrak{X}$ such that $F(\omega)=\operatorname{cl}\left\{f_{n}(\omega)\right\}$ for all $\omega \in \Omega$. Such a sequence $\left\{f_{n}\right\}$ is called a Castaing representation of $F$. If $F: \Omega \rightarrow \mathscr{K}(\mathfrak{X})$ is measurable, then the graph $\operatorname{Gr}(F)=\{(\omega, x) \in \Omega \times \mathfrak{X}: x \in F(\omega)\}$ of $F$ is $\mathscr{A} \otimes \mathscr{B}_{\mathfrak{X}^{-}}$ measurable. Also the converse holds if $(\Omega, \mathscr{A}, \mu)$ is complete. The proofs of these results are found in $[\mathbf{7}, \mathbf{1 8}]$. We denote by $\mathscr{M}[\Omega ; \mathscr{K}(\mathfrak{X})]$ the family of all multivalued random variables $F: \Omega \rightarrow \mathscr{K}(\mathfrak{X})$. 
For $1 \leqslant p<\infty, L^{p}(\Omega, \mathscr{A}, \mu ; \mathfrak{X})=L^{p}(\Omega ; \mathfrak{X})$ denotes the Banach space of (equivalence classes of) measurable functions $f: \Omega \rightarrow \mathfrak{X}$ such that the norm $\|f\|_{p}=$ $E\left(\|f\|^{p}\right)^{1 / p}=\left(\int_{\Omega}\|f(\omega)\|^{p} d \mu\right)^{1 / p}$ is finite, and $L^{p}(\Omega ; \mathbf{R})$ is denoted by $L^{p}$. For $F \in \mathscr{M}[\Omega ; \mathscr{K}(\mathfrak{X})]$, let

$$
S_{F}^{1}=\left\{f \in L^{1}(\Omega ; \mathfrak{X}): f(\omega) \in F(\omega) \text { a.s. }\right\}
$$

which is a closed subset of $L^{1}(\Omega ; \mathfrak{X})$ and is nonempty if and only if $d(0, F(\cdot))=$ $\inf _{x \in F(\cdot)}\|x\|$ is in $L^{1}$. If $S_{F}^{1} \neq \varnothing$, then there is a Castaing representation of $F$ contained in $S_{F}^{1}$ (cf. [16, Lemma 1.1]). The integral or mean $E[F]$ of $F$ is defined by

$$
E[F]=\int_{\Omega} F d \mu=\left\{E(f)=\int_{\Omega} f d \mu: f \in S_{F}^{1}\right\},
$$

where $E(f)=\int_{\Omega} f d \mu$ is the usual Bochner integral. This multivalued integral was introduced by Aumann [5]. For $A \in \mathscr{A}$, let $\int_{A} F d \mu$ be the integral of $F$ restricted on A.

Given a sub- $\sigma$-field $\mathscr{B}$ of $\mathscr{A}$ and a $\mathscr{B}$-measurable $F \in \mathscr{M}[\Omega ; \mathscr{K}(\mathfrak{X})]$, besides $S_{F}^{1}$ and $E[F]$ taken on $(\Omega, \mathscr{A}, \mu)$, we define on $(\Omega, \mathscr{B}, \mu)$

$$
\begin{gathered}
S_{F}^{1}(\mathscr{B})=\left\{f \in L^{1}(\Omega, \mathscr{B}, \mu ; \mathfrak{X}): f(\omega) \in F(\omega) \text { a.s. }\right\}, \\
E[F, \mathscr{B}]=\int_{\Omega}^{(\mathscr{B})} F d \mu=\left\{E(f): f \in S_{F}^{1}(\mathscr{B})\right\} .
\end{gathered}
$$

For $f \in L^{1}(\Omega ; \mathfrak{X})$, the conditional expectation of $f$ relative to $\mathscr{B}$ is given as a function $E(f \mid \mathscr{B}) \in L^{1}(\Omega, \mathscr{B}, \mu ; \mathfrak{X})$ such that $\int_{B} E(f \mid \mathscr{B}) d \mu=\int_{B} f d \mu$ for all $B \in \mathscr{B}$. If $F \in$ $\mathscr{M}[\Omega ; \mathscr{K}(\mathfrak{X})]$ with $S_{F}^{1} \neq \varnothing$, then it is seen (cf. [16, Theorem 5.1]) that there exists a unique (in the a.s. sense) $\mathscr{B}$-measurable $\mathscr{E}[F \mid \mathscr{B}] \in \mathscr{M}[\Omega ; \mathscr{K}(\mathfrak{X})]$ satisfying

$$
S_{\mathscr{E}[F \mid \mathscr{B}]}^{1}(\mathscr{B})=\operatorname{cl}\left\{E(f \mid \mathscr{B}): f \in S_{F}^{1}\right\}, \quad \text { the closure in } L^{1}(\Omega ; \mathfrak{X}) \text {. }
$$

We call $\mathscr{E}[F \mid \mathscr{B}]$ the (multivalued) conditional expectation of $F$ relative to $\mathscr{B}$. This conditional expectation $\mathscr{E}[F \mid \mathscr{B}]$ has the properties analogous to those of the usual conditional expectation (see $[16, \S 5])$. For example, we have

$$
\operatorname{cl} \int_{B}^{(\mathscr{B})} \mathscr{E}[F \mid \mathscr{B}] d \mu=\operatorname{cl} \int_{B} F d \mu, \quad B \in \mathscr{B},
$$

and if $F(\omega) \in \mathscr{K}_{c}(\mathfrak{X})$ a.s., then

$$
\operatorname{cl} \int_{B} \mathscr{E}[F \mid \mathscr{B}] d \mu=\operatorname{cl} \int_{B} F d \mu, \quad B \in \mathscr{B} .
$$

Though we assumed in [16] that $F \in \mathscr{M}[\Omega ; \mathscr{K}(\mathfrak{X})]$ is integrably bounded, i.e., $\|F(\cdot)\|=\sup _{x \in F(\cdot)}\|x\|$ is in $L^{1}$, most of the results and the proofs there remain valid for $F \in \mathscr{M}[\Omega ; \mathscr{K}(\mathfrak{X})]$ with $S_{F}^{1} \neq \varnothing$. Note that $\mathscr{E}[F \mid \mathscr{B}](\omega) \equiv \operatorname{cl} E[F]$ when $\mathscr{B}=$ $\{\varnothing, \Omega\}$. We refer to $[7,9,16,18]$ for detailed expositions on multivalued random variables and their integrals and conditional expectations.

Let $\left\{X_{n}\right\}$ be a sequence in $\mathscr{K}(\mathfrak{X})$. We write $X_{n} \stackrel{h}{\rightarrow} X$ if $h\left(X_{n}, X\right) \rightarrow 0$ for some $X \in \mathscr{K}(\mathfrak{X})$. Rather than this Hausdorff distance convergence, we use in this paper another notion of convergence introduced by Mosco [22, 23] following the Kuratowski convergence (cf. [21, p. 339]). Let s-lim inf $X_{n}$ be the set of all $x \in \mathfrak{X}$ such that $\left\|x_{n}-x\right\| \rightarrow 0$ for some $x_{n} \in X_{n}$, i.e., $d\left(x, X_{n}\right) \rightarrow 0$, and w-lim sup $X_{n}$ be the set of all $x \in \mathfrak{X}$ such that $x_{k} \stackrel{\text { w }}{\rightarrow} x$ (i.e., $x_{k}$ converges weakly to $x$ ) for some $x_{k} \in X_{n_{k}}$ and 
some subsequence $\left\{X_{n_{k}}\right\}$ of $\left\{X_{n}\right\}$. Clearly s-lim inf $X_{n} \subset$ w-lim sup $X_{n}$ holds, and s-lim $\inf X_{n} \in \mathscr{K}(\mathfrak{X})\left(\in \mathscr{K}_{c}(\mathfrak{X})\right.$ if $\left.\left\{X_{n}\right\} \subset \mathscr{K}_{c}(\mathfrak{X})\right)$ unless it is empty. We write simply $X_{n} \rightarrow X$ if

$$
\text { s-lim inf } X_{n}=X=\mathrm{w}-\lim \sup X_{n} \text {. }
$$

Concerning the Mosco convergence, see also Salinetti and Wets [26, 27] and Tsukada $[31,32]$.

We give here some elementary facts on w-lim sup $X_{n}$ for later use.

LEMMA 1.1. Let $\left\{X_{n}\right\}$ and $\left\{Y_{n}\right\}$ be sequences in $\mathscr{K}(\mathfrak{X})$.

(1) If $X \in \mathscr{K}_{c}(\mathfrak{X})$ and $\lim \sup s\left(X_{n}, x^{*}\right) \leqslant s\left(X, x^{*}\right)$ for every $x^{*} \in \mathfrak{X}^{*}$, then w-lim sup $X_{n} \subset X$.

Suppose that $\mathfrak{X}$ is reflexive. Then:

(2) If $\sup \left\|X_{n}\right\|<\infty$, then w-lim sup $X_{n}$ is nonempty weakly compact and

$$
\lim \sup s\left(X_{n}, x^{*}\right) \leqslant s\left(\mathrm{w}-\lim \sup X_{n}, x^{*}\right), \quad x^{*} \in \mathfrak{X}^{*} .
$$

(3) If $\sup \left\|X_{n}\right\|<\infty$, then

$$
\text { w-lim sup } \operatorname{cl}\left(X_{n}+Y_{n}\right) \subset \mathrm{w}-\lim \sup X_{n}+\mathrm{w}-\lim \sup Y_{n} \text {. }
$$

(4) If $\mathrm{w}-\lim \sup X_{n}$ and $\mathrm{w}-\lim \sup Y_{n}$ are nonempty, then

$$
h\left(\operatorname{cl}\left(\mathrm{w}-\lim \sup X_{n}\right), \operatorname{cl}\left(\mathrm{w}-\lim \sup Y_{n}\right)\right) \leqslant \lim \sup h\left(X_{n}, Y_{n}\right) \text {. }
$$

Proof. (1) If $x \in \mathrm{w}-\lim \sup X_{n}$, then $x_{k} \stackrel{\mathrm{w}}{\rightarrow} x$ for some $x_{k} \in X_{n_{k}}$ and hence

$$
\begin{aligned}
\left\langle x, x^{*}\right\rangle & =\lim \left\langle x_{k}, x^{*}\right\rangle \leqslant \lim \sup s\left(X_{n}, x^{*}\right) \\
& \leqslant s\left(X, x^{*}\right), \quad x^{*} \in \mathfrak{X}^{*},
\end{aligned}
$$

which implies $x \in X$.

(2) Let $r=\sup \left\|X_{n}\right\|<\infty$. Since $\{x \in \mathfrak{X}:\|x\| \leqslant r\}$ is compact and metrizable in the weak topology, we have

$$
\mathrm{w}-\lim \sup X_{n}=\bigcap_{m=1}^{\infty} \mathrm{w}-\mathrm{cl}\left(\bigcup_{n=m}^{\infty} X_{n}\right) \neq \varnothing \text {. }
$$

Given $x^{*} \in \mathfrak{X}^{*}$, a sequence $\left\{x_{k}\right\}$ of $x_{k} \in X_{n_{k}}$ can be chosen so that $\left\langle x_{k}, x^{*}\right\rangle \rightarrow$ $\lim \sup s\left(X_{n}, x^{*}\right)$ and $x_{k} \stackrel{\mathrm{w}}{\rightarrow} x$ for some $x \in \mathfrak{X}$. Hence $x \in \mathrm{w}-\lim \sup X_{n}$ and

$$
\lim \sup s\left(X_{n}, x^{*}\right)=\left\langle x, x^{*}\right\rangle \leqslant s\left(\mathrm{w}-\lim \sup X_{n}, x^{*}\right) \text {. }
$$

(3) If $z \in \mathrm{w}-\lim \sup \mathrm{cl}\left(X_{n}+Y_{n}\right)$, then $x_{k}+y_{k} \stackrel{\mathrm{w}}{\rightarrow} z$ with $x_{k} \in X_{n_{k}}$ and $y_{k} \in Y_{n_{k}}$. We may assume $x_{k} \stackrel{\mathrm{w}}{\rightarrow} x$ and so $y_{k} \stackrel{\mathrm{w}}{\rightarrow} y=z-x$. Hence $z=x+y \in \mathrm{w}-\lim \sup X_{n}$ $+\mathrm{w}-\lim \sup Y_{n}$.

(4) Assume $\lim \sup h\left(X_{n}, Y_{n}\right)<\infty$. Let $x \in \mathrm{w}-\lim \sup X_{n}$, i.e., $x_{k} \stackrel{\mathrm{w}}{\rightarrow} x$ with $x_{k} \in X_{n_{k}}$. For each $k \geqslant 1$, we select a $y_{k} \in Y_{n_{k}}$ such that $\left\|x_{k}-y_{k}\right\| \leqslant h\left(X_{n_{k}}, Y_{n_{k}}\right)+$ $k^{-1}$. Since $\left\{x_{k}\right\}$ and hence $\left\{y_{k}\right\}$ are bounded, assuming $y_{k} \stackrel{w}{\rightarrow} y$ we have $y \in$ w-lim $\sup Y_{n}$ and

$$
\begin{aligned}
d\left(x, \operatorname{cl}\left(w-\lim \sup Y_{n}\right)\right) & \leqslant\|x-y\| \leqslant \lim \inf \left\|x_{k}-y_{k}\right\| \\
& \leqslant \lim \sup h\left(X_{n}, Y_{n}\right) .
\end{aligned}
$$

Thus (4) is proved. Q.E.D. 
2. Convergence of multivalued conditional expectations. In this section, we establish several convergence theorems for multivalued conditional expectations (particularly for multivalued integrals). We notice that the proofs of convergence theorems in $[1,5,17,29]$ for multivalued integrals are essentially dependent on the finite dimensionality.

Throughout this section, let $\mathscr{B}$ be a fixed sub- $\sigma$-field of $\mathscr{A}$ and $\left\{F_{n}\right\}$ a sequence in $\mathscr{M}[\Omega ; \mathscr{K}(\mathfrak{X})]$. We first show the monotone convergence theorem. The same result for multivalued integrals was given in [14].

THEOREM 2.1. Suppose that $F_{1}(\omega) \subset F_{2}(\omega) \subset \cdots$ a.s. with $S_{F_{1}}^{1} \neq \varnothing$ and let $F(\omega)$ $=\operatorname{cl}\left(\bigcup_{n=1}^{\infty} F_{n}(\omega)\right), \omega \in \Omega$. Then $F \in \mathscr{M}[\Omega ; \mathscr{K}(\mathfrak{X})]$ and

$$
\mathscr{E}[F \mid \mathscr{B}](\omega)=\operatorname{cl}\left(\bigcup_{n=1}^{\infty} \mathscr{E}\left[F_{n} \mid \mathscr{B}\right](\omega)\right) \text { a.s. }
$$

Proof. Let $G(\omega)=\operatorname{cl}\left(\bigcup_{n=1}^{\infty} \mathscr{E}\left[F_{n} \mid \mathscr{B}\right](\omega)\right), \omega \in \Omega$. Then $F, G \in \mathscr{M}[\Omega ; \mathscr{K}(\mathfrak{X})]$ and $G$ is $\mathscr{B}$-measurable. Obviously,

$$
\begin{gathered}
S_{F_{1}}^{1} \subset S_{F_{2}}^{1} \subset \cdots \subset S_{F}^{1}, \\
S_{\mathscr{E}\left[F_{1} \mid \mathscr{B}\right]}^{1}(\mathscr{B}) \subset S_{\mathscr{E}\left[F_{2} \mid \mathscr{B}\right]}^{1}(\mathscr{B}) \subset \cdots \subset S_{G}^{1}(\mathscr{B}) .
\end{gathered}
$$

For any $f \in S_{F}^{1}$, using [16, Theorem 2.2] we have

$$
\inf _{g \in S_{F_{n}}^{1}}\|f-g\|_{1}=E\left(d\left(f(\cdot), F_{n}(\cdot)\right)\right) \rightarrow 0
$$

since $d\left(f(\cdot), F_{n}(\cdot)\right) \in L^{1}$ and $d\left(f(\omega), F_{n}(\omega)\right) \downarrow 0$ a.s. Hence $S_{F}^{1}=\operatorname{cl}\left(\cup_{n=1}^{\infty} S_{F_{n}}^{1}\right)$ and similarly $S_{G}^{1}(\mathscr{B})=\operatorname{cl}\left(\bigcup_{n=1}^{\infty} S_{\mathscr{E}\left[F_{n} \mid \mathscr{B}\right]}^{1}(\mathscr{B})\right)$. Thus

$$
S_{\mathscr{E}[F \mid \mathscr{B}]}^{1}(\mathscr{B})=\mathrm{cl}\left(\bigcup_{n=1}^{\infty}\left\{E(f \mid \mathscr{B}): f \in S_{F_{n}}^{1}\right\}\right)=S_{G}^{1}(\mathscr{B}),
$$

which implies $\mathscr{E}[F \mid \mathscr{B}](\omega)=G(\omega)$ a.s. Q.E.D.

Before giving Fatou's lemmas, we state the following theorem concerning the measurability of s-lim inf and w-lim sup of $\left\{F_{n}\right\}$ as the infinite dimensional extension of [28, Theorem 3.1].

THEOREM 2.2. (1) If s-liminf $F_{n}(\omega) \neq \varnothing$ a.s., there exists an $F \in \mathscr{M}[\Omega ; \mathscr{K}(\mathfrak{X})]$ such that

$$
F(\omega)=\operatorname{s-lim} \inf F_{n}(\omega) \text { a.s. }
$$

(2) Suppose that $\mathfrak{X}$ is reflexive. If $\sup \left\|F_{n}(\omega)\right\|<\infty$ a.s., then there exists an $F \in \mathscr{M}[\Omega ; \mathscr{K}(\mathfrak{X})]$ such that

$$
F(\omega)=\mathrm{w}-\lim \sup F_{n}(\omega) \text { a.s. }
$$

Proof. (1) Let $G(\omega)=$ s-lim inf $F_{n}(\omega)$. We may assume that $G(\omega) \neq \varnothing$ for all $\omega \in \Omega$. Since

$$
\operatorname{Gr}(G)=\left\{(\omega, x) \in \Omega \times \mathfrak{X}: d\left(x, F_{n}(\omega)\right) \rightarrow 0\right\} \in \mathscr{A} \otimes \mathscr{B}_{\mathfrak{X}},
$$


there is a sequence $\left\{g_{n}\right\}$ of $\overline{\mathscr{A}}$-measurable functions $g_{n}: \Omega \rightarrow \mathfrak{X}$ such that $G(\omega)=$ $\operatorname{cl}\left\{g_{n}(\omega)\right\}$ for all $\omega \in \Omega$, where $\overline{\mathscr{A}}$ is the completion of $\mathscr{A}$ with respect to $\mu$. Choosing $\mathscr{A}$-measurable functions $f_{n}: \Omega \rightarrow \mathfrak{X}$ with $f_{n}(\omega)=g_{n}(\omega)$ a.s., we define $F(\omega)=$ $\operatorname{cl}\left\{f_{n}(\omega)\right\}, \omega \in \Omega$. Then $F \in \mathscr{M}[\Omega ; \mathscr{K}(\mathfrak{X})]$ and $F(\omega)=G(\omega)$ a.s.

(2) We may assume that $\left\|F_{n}(\omega)\right\| \leqslant r(<\infty)$ for all $\omega \in \Omega$ and $n \geqslant 1$. Let $\mathfrak{X}_{r}=\{x \in \mathfrak{X}:\|x\| \leqslant r\}$ which is a compact metric space in the weak topology. $\mathscr{C}_{w}\left(\mathfrak{X}_{r}\right)$ denotes the family of all nonempty weakly closed (i.e., weakly compact) subsets of $\mathfrak{X}_{r}$. Now let $F(\omega)=\mathrm{w}-\lim \sup F_{n}(\omega)$ and $G_{m}(\omega)=\mathrm{w}-\operatorname{cl}\left(\bigcup_{n=m}^{\infty} F_{n}(\omega)\right)$, $m \geqslant 1$. We then have $F(\omega)=\bigcap_{m=1}^{\infty} G_{m}(\omega) \neq \varnothing$ for all $\omega \in \Omega$. Since each $G_{m}$ is measurable as a multivalued function $G_{m}: \Omega \rightarrow \mathscr{C}_{w}\left(\mathfrak{X}_{r}\right), F$ is measurable as $F$ : $\Omega \rightarrow \mathscr{C}_{w}\left(\mathfrak{X}_{r}\right)$ by [7, Proposition III.4]. Hence it is seen from [7, Theorem II-10] that

$$
F^{-}(C)=\Omega \backslash\left\{\omega \in \Omega: F(\omega) \subset \mathfrak{X}_{r} \backslash C\right\} \in \mathscr{A}
$$

for every $C \in \mathscr{C}_{w}\left(\mathfrak{X}_{r}\right)$. For each open $O \subset \mathfrak{X}$, taking a sequence $\left\{V_{j}\right\}$ of closed balls with $O=\bigcup_{j=1}^{\infty} V_{j}$, we have

$$
F^{-}(O)=\bigcup_{j=1}^{\infty} F^{-}\left(\mathfrak{X}_{r} \cap V_{j}\right) \in \mathscr{A} .
$$

Thus $F \in \mathscr{M}[\Omega ; \mathscr{K}(\mathfrak{X})]$. Q.E.D.

REMARK. If $\sup \left\|F_{n}(\omega)\right\|<\infty$ for every $\omega \in \Omega$ in Theorem 2.2(2), then the above proof shows that w-lim $\sup F_{n}(\cdot)$ itself is in $\mathscr{M}[\Omega ; \mathscr{K}(\mathfrak{X})]$. This remark, together with the improvement of the above proof (2), is due to M. Valadier.

In the subsequent theorems, we always assume that $F \in \mathscr{M}[\Omega ; \mathscr{K}(\mathfrak{X})]$. We now prove two types of Fatou's lemmas.

THEOREM 2.3. Suppose that there exists $a \xi \in L^{1}$ with $d\left(0, F_{n}(\omega)\right) \leqslant \xi(\omega)$ a.s. for all $n \geqslant 1$. If $F(\omega)=$ s-lim inf $F_{n}(\omega)$ a.s. and $S_{F}^{1} \neq \varnothing$, then

$$
\mathscr{E}[F \mid \mathscr{B}](\omega) \subset \mathrm{s}-\lim \inf \mathscr{E}\left[F_{n} \mid \mathscr{B}\right](\omega) \text { a.s. }
$$

Proof. For each $f \in S_{F}^{1}$ and $n \geqslant 1$, define $G_{n}: \Omega \rightarrow \mathscr{K}(\mathfrak{X})$ by

$$
G_{n}(\omega)=\left\{x \in F_{n}(\omega):\|f(\omega)-x\| \leqslant d\left(f(\omega), F_{n}(\omega)\right)+n^{-1}\right\}, \quad \omega \in \Omega .
$$

Since $\operatorname{Gr}\left(G_{n}\right) \in \mathscr{A} \otimes \mathscr{B}_{\mathfrak{X}}$, we can select a measurable function $f_{n}: \Omega \rightarrow \mathfrak{X}$ with $f_{n}(\omega) \in G_{n}(\omega)$ a.s. Since $d\left(f(\omega), F_{n}(\omega)\right) \rightarrow 0$ a.s. and

$$
\begin{aligned}
\left\|f(\omega)-f_{n}(\omega)\right\| & \leqslant d\left(f(\omega), F_{n}(\omega)\right)+n^{-1} \\
& \leqslant\|f(\omega)\|+\xi(\omega)+1 \text { a.s. }
\end{aligned}
$$

we have $f_{n} \in S_{F_{n}}^{1}$ and $\left\|f(\omega)-f_{n}(\omega)\right\| \rightarrow 0$ a.s. By Lebesgue's convergence theorem for usual conditional expectations, it follows that

$$
\begin{aligned}
d\left(E(f \mid \mathscr{B})(\omega), \mathscr{E}\left[F_{n} \mid \mathscr{B}\right](\omega)\right) & \leqslant\left\|E(f \mid \mathscr{B})(\omega)-E\left(f_{n} \mid \mathscr{B}\right)(\omega)\right\| \\
& \leqslant E\left(\left\|f(\cdot)-f_{n}(\cdot)\right\| \mid \mathscr{B}\right)(\omega) \rightarrow 0 \quad \text { a.s. }
\end{aligned}
$$

and hence

$$
E(f \mid \mathscr{B})(\omega) \in \operatorname{s-lim} \inf \mathscr{E}\left[F_{n} \mid \mathscr{B}\right](\omega) \text { a.s. }
$$


For any $g \in S_{\mathscr{E}[F \mid \mathscr{B}]}^{1}(\mathscr{B})$, there is a sequence $\left\{f_{j}\right\}$ in $S_{F}^{1}$ with $\left\|E\left(f_{j} \mid \mathscr{B}\right)(\omega)-g(\omega)\right\|$ $\rightarrow 0$ a.s., so that $g(\omega) \in \operatorname{s-lim} \inf \mathscr{E}\left[F_{n} \mid \mathscr{B}\right](\omega)$ a.s. Taking a Castaing representation of $\mathscr{E}[F \mid \mathscr{B}]$ contained in $S_{\mathscr{E}[F \mid \mathscr{B}]}^{1}(\mathscr{B})$, we obtain the desired conclusion. Q.E.D.

THEOREM 2.4. Suppose that $\mathfrak{X}$ is reflexive and there exists $a \xi \in L^{1}$ with $\left\|F_{n}(\omega)\right\| \leqslant$ $\xi(\omega)$ a.s. for all $n \geqslant 1$. If $F(\omega)=\mathrm{w}-\lim \sup F_{n}(\omega)$ a.s., then

$$
\text { w-lim } \sup \mathscr{E}\left[F_{n} \mid \mathscr{B}\right](\omega) \subset \mathscr{E}[\overline{\operatorname{co}} F \mid \mathscr{B}](\omega) \text { a.s., }
$$

where $\overline{\mathrm{co}} F \in \mathscr{M}[\Omega ; \mathscr{K}(\mathfrak{X})]$ is given by $(\overline{\mathrm{co}} F)(\omega)=\overline{\mathrm{co}} F(\omega)$.

Proof. We get also $\|F(\omega)\| \leqslant \xi(\omega)$ a.s. Let $x^{*} \in \mathfrak{X}^{*}$, then $s\left(F(\cdot), x^{*}\right) \in L^{1}$. Using [16, Theorem 2.2], we have

$$
\begin{aligned}
\int_{B} E\left(s\left(F(\cdot), x^{*}\right) \mid \mathscr{B}\right)(\omega) d \mu & =\int_{B} s\left(F(\omega), x^{*}\right) d \mu=\sup _{f \in S_{F}^{1}} \int_{B}\left\langle f(\omega), x^{*}\right\rangle d \mu \\
& =\sup _{f \in S_{F}^{1}} \int_{B}\left\langle E(f \mid \mathscr{B})(\omega), x^{*}\right\rangle d \mu \\
& =\sup _{g \in S_{\mathscr{E}}^{1}[F \mid \mathscr{B}]} \int_{B}\left\langle g(\omega), x^{*}\right\rangle d \mu \\
& =\int_{B} s\left(\mathscr{E}[F \mid \mathscr{B}](\omega), x^{*}\right) d \mu, \quad B \in \mathscr{B} .
\end{aligned}
$$

Hence

$$
E\left(s\left(F(\cdot), x^{*}\right) \mid \mathscr{B}\right)(\omega)=s\left(\mathscr{E}[F \mid \mathscr{B}](\omega), x^{*}\right) \text { a.s. }
$$

and similarly

$$
E\left(s\left(F_{n}(\cdot), x^{*}\right) \mid \mathscr{B}\right)(\omega)=s\left(\mathscr{E}\left[F_{n} \mid \mathscr{B}\right](\omega), x^{*}\right) \quad \text { a.s., } \quad n \geqslant 1 .
$$

Further, by [16, Theorem 5.2(4 $\left.4^{\circ}\right)$,

$$
\mathscr{E}[\overline{\operatorname{co}} F \mid \mathscr{B}](\omega)=\overline{\operatorname{co}} \mathscr{E}[F \mid \mathscr{B}](\omega) \text { a.s. }
$$

Thus, by Fatou's lemma for usual conditional expectations and Lemma 1.1(2), we have

$$
\begin{aligned}
& \lim \sup s\left(\mathscr{E}\left[F_{n} \mid \mathscr{B}\right](\omega), x^{*}\right) \leqslant E\left(\lim \sup s\left(F_{n}(\cdot), x^{*}\right) \mid \mathscr{B}\right)(\omega) \\
& \leqslant E\left(s\left(F(\cdot), x^{*}\right) \mid \mathscr{B}\right)(\omega)=s\left(\mathscr{E}[\overline{\operatorname{co}} F \mid \mathscr{B}](\omega), x^{*}\right) \text { a.s. }
\end{aligned}
$$

Because of the separability of $\mathfrak{X}^{*}$, there exists a $\mu$-null set $N \in \mathscr{A}$ such that

$$
\lim \sup s\left(\mathscr{E}\left[F_{n} \mid \mathscr{B}\right](\omega), x^{*}\right) \leqslant s\left(\mathscr{E}[\overline{\operatorname{co}} F \mid \mathscr{B}](\omega), x^{*}\right), \quad x^{*} \in \mathfrak{X}^{*}, \omega \in \Omega \backslash N .
$$

This shows the theorem from Lemma 1.1(1). Q.E.D.

An $A \in \mathscr{A}$ is called a $\mathscr{B}$-atom if for each $A^{\prime} \in \mathscr{A}$ with $A^{\prime} \subset A$, there exists a $B \in \mathscr{B}$ satisfying $\mu\left((A \cap B) \Delta A^{\prime}\right)=0$. According to Valadier [33], if $(\Omega, \mathscr{A}, \mu)$ has no $\mathscr{B}$-atom, then

$$
\mathscr{E}[\overline{\operatorname{co}} F \mid \mathscr{B}](\omega)=\mathscr{E}[F \mid \mathscr{B}](\omega) \text { a.s. }
$$

for every $F \in \mathscr{M}[\Omega ; \mathscr{K}(\mathfrak{X})]$ with $S_{F}^{1} \neq \varnothing$. 
By Theorems 2.3 and 2.4 and the result stated just now, we obtain the following Lebesgue's convergence theorem.

THEOREM 2.5. Suppose that $\mathfrak{X}$ is reflexive and there exists $a \xi \in L^{1}$ with $\left\|F_{n}(\omega)\right\| \leqslant$ $\xi(\omega)$ a.s. for all $n \geqslant 1$. If $F_{n}(\omega) \rightarrow F(\omega)$ a.s. and if $(\Omega, \mathscr{A}, \mu)$ has no $\mathscr{B}$-atom or $F(\omega) \in \mathscr{K}_{c}(\mathfrak{X})$ a.s. (this is the case when $F_{n}(\omega) \in \mathscr{K}_{c}(\mathfrak{X})$ a.s. for all $n \geqslant 1$ ), then

$$
\mathscr{E}\left[F_{n} \mid \mathscr{B}\right](\omega) \rightarrow \mathscr{E}[F \mid \mathscr{B}](\omega) \text { a.s. }
$$

To prove Lebesgue's convergence theorem in the Hausdorff distance, we need

LEMMA 2.6. For each integrably bounded $F, G \in \mathscr{M}[\Omega ; \mathscr{K}(\mathfrak{X})]$,

$$
h(\mathscr{E}[F \mid \mathscr{B}](\omega), \mathscr{E}[G \mid \mathscr{B}](\omega)) \leqslant E(h(F(\cdot), G(\cdot)) \mid \mathscr{B})(\omega) \text { a.s. }
$$

Proof. Since $h(F(\omega), G(\omega)) \leqslant\|F(\omega)\|+\|G(\omega)\|$, we get $h(F(\cdot), G(\cdot)) \in L^{1}$. As is seen from the last part of the proof of Theorem 2.3, there exists a sequence $\left\{f_{n}\right\}$ in $S_{F}^{1}$ such that $\mathscr{E}[F \mid \mathscr{B}](\omega)=\operatorname{cl}\left\{E\left(f_{n} \mid \mathscr{B}\right)(\omega)\right\}$ a.s. For each $n \geqslant 1$, we select a sequence $\left\{g_{n j}: j \geqslant 1\right\}$ in $S_{G}^{1}$ such that $\left\|f_{n}(\omega)-g_{n j}(\omega)\right\| \downarrow d\left(f_{n}(\omega), G(\omega)\right)$ a.s. as $j \rightarrow \infty$. We then have

$$
\begin{aligned}
\sup _{x \in \mathscr{E}[F \mid \mathscr{B}](\omega)} d(x, \mathscr{E}[G \mid \mathscr{B}](\omega)) & \leqslant \sup _{n} \inf _{j}\left\|E\left(f_{n} \mid \mathscr{B}\right)(\omega)-E\left(g_{n j} \mid \mathscr{B}\right)(\omega)\right\| \\
& \leqslant \sup _{n} \inf _{j} E\left(\left\|f_{n}(\cdot)-g_{n j}(\cdot)\right\| \mid \mathscr{B}\right)(\omega) \\
& =\sup _{n} E\left(d\left(f_{n}(\cdot), G(\cdot)\right) \mid \mathscr{B}\right)(\omega) \\
& \leqslant E\left(\sup _{n} d\left(f_{n}(\cdot), G(\cdot)\right) \mid \mathscr{B}\right)(\omega) \\
& \leqslant E(h(F(\cdot), G(\cdot)) \mid \mathscr{B})(\omega) \text { a.s. }
\end{aligned}
$$

Thus the lemma is proved. Q.E.D.

We write $F_{n} \stackrel{h}{\rightarrow} F$ in probability if $h\left(F_{n}(\cdot), F(\cdot)\right) \rightarrow 0$ in probability.

THEOREM 2.7. Suppose that there exists $a \xi \in L^{1}$ with $\left\|F_{n}(\omega)\right\| \leqslant \xi(\omega)$ a.s. for all $n \geqslant 1$. If $F_{n}(\omega) \stackrel{h}{\rightarrow} F(\omega)$ a.s. (resp. $F_{n} \stackrel{h}{\rightarrow} F$ in probability), then

$$
\begin{aligned}
\mathscr{E}\left[F_{n} \mid \mathscr{B}\right](\omega) \stackrel{h}{\rightarrow} \mathscr{E}[F \mid \mathscr{B}](\omega) \text { a.s. } \\
\left(\text { resp. } \mathscr{E}\left[F_{n} \mid \mathscr{B}\right] \stackrel{h}{\rightarrow} \mathscr{E}[F \mid \mathscr{B}] \text { in probability }\right) .
\end{aligned}
$$

Proof. Since $h\left(F_{n}(\omega), F(\omega)\right) \leqslant 2 \xi(\omega)$ a.s. and $h\left(F_{n}(\omega), F(\omega)\right) \rightarrow 0$ a.s., by Lemma 2.6 we have

$$
h\left(\mathscr{E}\left[F_{n} \mid \mathscr{B}\right](\omega), \mathscr{E}[F \mid \mathscr{B}](\omega)\right) \leqslant E\left(h\left(F_{n}(\cdot), F(\cdot)\right) \mid \mathscr{B}\right)(\omega) \rightarrow 0 \quad \text { a.s. }
$$

The assertion for the convergence in probability is verified by the argument of selecting a.s. convergent subsequence. Q.E.D.

Particularly when $\mathscr{B}=\{\varnothing, \Omega\}$, the above theorems give the corresponding convergence theorems for multivalued integrals. Indeed we obtain more than those as follows. 
THEOREM 2.8. (1) Suppose that $\left\{d\left(0, F_{n}(\cdot)\right)\right\}$ is uniformly integrable. If $F(\omega)=$ s-lim inf $F_{n}(\omega)$ a.s. and $S_{F}^{1} \neq \varnothing$, then

$$
\operatorname{cl} E[F] \subset \mathrm{s}-\lim \inf \mathrm{cl} E\left[F_{n}\right] .
$$

(2) Suppose that $\mathfrak{X}$ is reflexive and $\left\{\left\|F_{n}(\cdot)\right\|\right\}$ is uniformly integrable. If $F(\omega)=$ $\mathrm{w}-\lim \sup F_{n}(\omega)$ a.s. and $S_{F}^{1} \neq \varnothing$, then

$$
\text { w-lim sup } \operatorname{cl} E\left[F_{n}\right] \subset \operatorname{cl} E[F] \text {. }
$$

(3) Suppose that $\mathfrak{X}$ is reflexive and $\left\{\left\|F_{n}(\cdot)\right\|\right\}$ is uniformly integrable. If $F_{n}(\omega) \rightarrow$ $F(\omega)$ a.s., then

$$
\mathrm{cl} E\left[F_{n}\right] \rightarrow \operatorname{cl} E[F] .
$$

(4) Suppose that $\left\{\left\|F_{n}(\cdot)\right\|\right\}$ is uniformly integrable. If $F_{n} \stackrel{h}{\rightarrow} F$ in probability, then

$$
\mathrm{cl} E\left[F_{n}\right] \stackrel{h}{\rightarrow} \mathrm{cl} E[F]
$$

ProOF. First recall that Fatou's lemma and Lebesgue's convergence theorem for usual integrals hold under the uniform integrability condition. So the proofs of (1) and (4) are analogous to those of Theorems 2.3 and 2.7.

(2) To prove this, it suffices in view of Lemma 1.1(3) to show the nonatomic case and the purely atomic case. If $(\Omega, \mathscr{A}, \mu)$ has no atom, then cl $E[F]$ is convex (cf. [16, Theorem 4.2]). Let $f \in S_{F}^{1}$ and define $F_{n j} \in \mathscr{M}[\Omega ; \mathscr{K}(\mathfrak{X})], n, j \geqslant 1$, by $F_{n j}(\omega)=$ $F_{n}(\omega)$ if $\left\|F_{n}(\omega)\right\| \leqslant j, F_{n j}(\omega)=\{f(\omega)\}$ otherwise. Since $\left\{\left\|F_{n}(\cdot)\right\|\right\}$ is uniformly integrable, we have

$$
\begin{aligned}
\sup _{n} h\left(\mathrm{cl} E\left[F_{n j}\right], \operatorname{cl} E\left[F_{n}\right]\right) \leqslant \sup _{n} E\left(h\left(F_{n j}(\cdot), F_{n}(\cdot)\right)\right) \\
\leqslant \sup _{n} \int_{\left\{\left\|F_{n}(\omega)\right\|>j\right\}}\left(\left\|F_{n}(\omega)\right\|+\|f(\omega)\|\right) d \mu \rightarrow 0 \quad \text { as } j \rightarrow \infty .
\end{aligned}
$$

Hence Lemma 1.1(4) implies

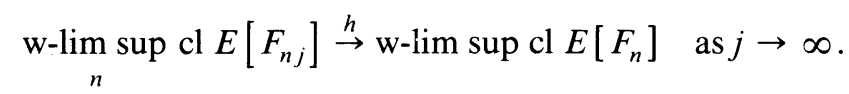

Moreover, for each $j \geqslant 1,\left\{\left\|F_{n j}(\cdot)\right\|: n \geqslant 1\right\}$ is uniformly integrable, $\sup _{n}\left\|F_{n j}(\omega)\right\|$ $<\infty$ for all $\omega \in \Omega$ and $\omega-\lim \sup _{n} F_{n j}(\omega) \subset F(\omega)$ a.s. By Lemma 1.1(2) and Theorem 2.2(2), we have

$$
\begin{aligned}
\limsup _{n}\left(\operatorname{cl} E\left[F_{n j}\right], x^{*}\right) & \leqslant E\left(\lim _{n} \sup s\left(F_{n j}(\cdot), x^{*}\right)\right) \\
& \leqslant E\left(s\left(\underset{n}{\operatorname{w}-\lim \sup } F_{n j}(\cdot), x^{*}\right)\right) \\
& \leqslant s\left(\operatorname{cl} E[F], x^{*}\right), \quad x^{*} \in \mathfrak{X}^{*}, j \geqslant 1,
\end{aligned}
$$

as in the proof of Theorem 2.4. Hence, by Lemma 1.1(1),

$$
\underset{n}{\mathrm{w}-\lim _{n} \sup \mathrm{cl} E\left[F_{n j}\right] \subset \operatorname{cl} E[F], \quad j \geqslant 1 .}
$$

Thus the desired conclusion follows from (2.1) and (2.2). 
If $(\Omega, \mathscr{A}, \mu)$ is purely atomic, then there are countable disjoint atoms $A_{j} \in \mathscr{A}$, $j \geqslant 1$, with $\Omega=\bigcup_{j} A_{j}$. Hence $F$ and $F_{n}$ are given by $F(\omega)=\sum_{j} 1_{A_{j}}(\omega) X_{j}$ and $F_{n}(\omega)=\sum_{j} 1_{A_{j}}(\omega) X_{n j}$, where $1_{A_{j}}$ is the characteristic function of $A_{j}$ and $X_{j}, X_{n j} \in$ $\mathscr{K}(\mathfrak{X})$ with $X_{j}=\mathrm{w}-\lim \sup _{n} X_{n j}$. Since $\left\{\left\|F_{n}(\cdot)\right\|\right\}$ is uniformly integrable, we have $\sup _{n}\left\|X_{n j}\right\|<\infty$ for all $j \geqslant 1$ and

$$
\sup _{n} h\left(\operatorname{cl} \sum_{j \leqslant m} \mu\left(A_{j}\right) X_{n j}, \operatorname{cl} E\left[F_{n}\right]\right) \leqslant \sup _{n} \sum_{j>m} \mu\left(A_{j}\right)\left\|X_{n j}\right\| \rightarrow 0 \quad \text { as } m \rightarrow \infty .
$$

Hence Lemma 1.1(4) implies

$$
\underset{n}{\mathrm{w}-\lim _{n} \sup \mathrm{cl}} \sum_{j \leqslant m} \mu\left(A_{j}\right) X_{n j} \stackrel{h}{\rightarrow} \text { w-lim sup cl } E\left[F_{n}\right] \quad \text { as } m \rightarrow \infty .
$$

Moreover it follows from Lemma 1.1(3) that

$$
\text { w-lim sup cl } \sum_{j \leqslant m} \mu\left(A_{j}\right) X_{n j} \subset \sum_{j \leqslant m} \mu\left(A_{j}\right) X_{j}, \quad m \geqslant 1 .
$$

Now let $x \in \mathrm{w}$-lim sup cl $E\left[F_{n}\right]$. Then, by (2.3) and (2.4), $\left\|x_{m}-x\right\| \rightarrow 0$ for some $x_{m} \in \sum_{j \leqslant m} \mu\left(A_{j}\right) X_{j}$. From $S_{F}^{1} \neq \varnothing$, we get $\sum_{j} \mu\left(A_{j}\right)\left\|y_{j}\right\|<\infty$ with $y_{j} \in X_{j}, j \geqslant 1$. Since

$$
\begin{gathered}
x_{m}+\sum_{j>m} \mu\left(A_{j}\right) y_{j} \in E[F], \\
\left\|x_{m}+\sum_{j>m} \mu\left(A_{j}\right) y_{j}-x\right\| \rightarrow 0 \text { as } m \rightarrow \infty,
\end{gathered}
$$

we obtain $x \in \operatorname{cl} E[F]$. Thus (2) is proved.

(3) Since $\|F(\omega)\| \leqslant \liminf \left\|F_{n}(\omega)\right\|$ a.s., we get $\|F(\cdot)\| \in L^{1}$. Hence (3) is immediate from (1) and (2). Q.E.D.

REMARK. It is obvious that the convergence theorems (1)-(4) in Theorems 2.8 for multivalued integrals are true on a $\sigma$-finite measure space $(\Omega, \mathscr{A}, \mu)$ when $\left\{d\left(0, F_{n}(\cdot)\right)\right\}$ (resp. $\left.\left\{\left\|F_{n}(\cdot)\right\|\right\}\right)$ is dominated by an $L^{1}$-function. Here the term a.s. is replaced by a.e. and (4) holds if $F_{n} \stackrel{h}{\rightarrow} F$ a.e. or in measure.

3. Multivalued strong laws of large numbers. There are two types of strong laws of large numbers. The first is the strong law for i.i.d. random variables, and the second is for independent random variables with the same mean and some $L^{p}$-norm condition. The first is generally valid for Banach space-valued random variables (cf. [24]). On the other hand, the second is not necessarily valid for the Banach space-valued case and is closely connected with some geometric conditions of a Banach space (see below). In this section, we obtain two types of strong laws of large numbers for multivalued random variables. The same strong laws were considered in [15] for weakly compact convex-valued random variables.

Given $F \in \mathscr{M}[\Omega ; \mathscr{K}(\mathfrak{X})]$, we define a sub- $\sigma$-field $\mathscr{A}_{F}$ of $\mathscr{A}$ by $\mathscr{A}_{F}=\left\{F^{-1}(\mathscr{U})\right.$ : $\left.\mathscr{U} \in \mathscr{B}_{\mathscr{K}(\mathfrak{X})}\right\}$, where $F^{-1}(\mathscr{U})=\{\omega \in \Omega: F(\omega) \in \mathscr{U}\}$, i.e., $\mathscr{A}_{F}$ is the smallest sub- $\sigma$ field of $\mathscr{A}$ with respect to which $F$ is measurable. The distribution of $F$ is a probability measure $\mu_{F}$ on $\mathscr{B}_{\mathscr{K}(\mathfrak{X})}$ defined by $\mu_{F}(\mathscr{U})=\mu\left(F^{-1}(\mathscr{U})\right), \mathscr{U} \in \mathscr{B}_{\mathscr{K}(\mathfrak{X})}$. Multivalued 
random variables $F_{i} \in \mathscr{M}[\Omega ; \mathscr{K}(\mathfrak{X})], i \in I$, are said to be independent if $\mathscr{A}_{F_{i}}, i \in I$, are independent, identically distributed if all $\mu_{F_{i}}$ are identical, and i.i.d. if they are independent and identically distributed.

In the following lemma, we state a part of the results in [14] (see also $[3,11]$ for the case $\mathfrak{X}=\mathbf{R}^{d}$ ). We give the short proof for convenience.

Lemma 3.1. (1) For each $F \in \mathscr{M}[\Omega ; \mathscr{K}(\mathfrak{X})]$ with $S_{F}^{1} \neq \varnothing$,

$$
\overline{\operatorname{co}} E[F]=\overline{\operatorname{co}} E\left[F, \mathscr{A}_{F}\right] \text {. }
$$

(2) Let $F, G \in \mathscr{M}[\Omega ; \mathscr{K}(\mathfrak{X})]$ be identically distributed. For each $f \in S_{F}^{1}\left(\mathscr{A}_{F}\right)$, there exists a $g \in S_{G}^{1}\left(\mathscr{A}_{G}\right)$ such that $f$ and $g$ are identically distributed.

(3) For each identically distributed $F, G \in \mathscr{M}[\Omega ; \mathscr{K}(\mathfrak{X})]$ with $S_{F}^{1} \neq \varnothing$,

$$
E\left[F, \mathscr{A}_{F}\right]=E\left[G, \mathscr{A}_{G}\right] .
$$

Proof. (1) Since $\overline{\text { co }} F$ is $\mathscr{A}_{F}$-measurable (cf. [16, Theorem 1.5]), by [16, (5.5)] we have

$$
S_{\overline{\mathrm{co}} F}^{1}\left(\mathscr{A}_{F}\right)=\left\{E\left(f \mid \mathscr{A}_{F}\right): f \in S_{\overline{\mathrm{co}} F}^{1}\right\} \text {. }
$$

Moreover $S_{\overline{\mathrm{co}} F}^{1}=\overline{\operatorname{co}} S_{F}^{1}$ and $S_{\overline{\mathrm{co}} F}^{1}\left(\mathscr{A}_{F}\right)=\overline{\operatorname{co}} S_{F}^{1}\left(\mathscr{A}_{F}\right)$ by [16, Theorem 1.5]. Hence

$$
\begin{aligned}
\overline{\operatorname{co}} E[F] & =\operatorname{cl} E[\overline{\operatorname{co}} F]=\operatorname{cl}\left\{E\left(E\left(f \mid \mathscr{A}_{F}\right)\right): f \in S_{\overline{\mathrm{co}} F}^{1}\right\} \\
& =\operatorname{cl}\left\{E(f): f \in S_{\overline{\mathrm{co}} F}^{1}\left(\mathscr{A}_{F}\right)\right\}=\overline{\operatorname{co}} E\left[F, \mathscr{A}_{F}\right] .
\end{aligned}
$$

(2) Since $\mathfrak{X}$ is separable and $f$ is $\mathscr{A}_{F}$-measurable, it is not hard to show that there exists a $\left(\mathscr{B}_{\mathscr{K}(\mathfrak{X})}, \mathscr{B}_{\mathfrak{X}}\right)$-measurable function $\Phi: \mathscr{K}(\mathfrak{X}) \rightarrow \mathfrak{X}$ satisfying $f(\omega)=\Phi(F(\omega))$ for every $\omega \in \Omega$. Now define $g(\omega)=\Phi(G(\omega)), \omega \in \Omega$. Since $F$ and $G$ are identically distributed, $f$ and $g$ are also. We have

$$
\int_{\Omega}\|g(\omega)\| d \mu=\int_{\mathscr{K}(\mathfrak{X})}\|\Phi(X)\| d \mu_{G}=\int_{\mathscr{K}(\mathfrak{X})}\|\Phi(X)\| d \mu_{F}=\int_{\Omega}\|f(\omega)\| d \mu<\infty .
$$

Because the function $(x, X) \mapsto d(x, X)$ of $\mathfrak{X} \times \mathscr{K}(\mathfrak{X})$ into $\mathbf{R}$ is $\mathscr{B}_{\mathfrak{X}} \otimes \mathscr{B}_{\mathscr{K}(\mathfrak{X})^{-}}$ measurable, $d(f(\cdot), F(\cdot))$ and $d(g(\cdot), G(\cdot))$ are identically distributed. Hence $d(f(\omega), F(\omega))=0$ a.s. implies $d(g(\omega), G(\omega))=0$ a.s. Thus $g \in S_{G}^{1}\left(\mathscr{A}_{G}\right)$ follows.

(3) is immediate from (2). Q.E.D.

REMARK. If $F(\omega) \in \mathscr{K}_{c}(\mathfrak{X})$ a.s. in Lemma 3.1(1), then $E[F]=E\left[F, \mathscr{A}_{F}\right]$ since $S_{F}^{1}\left(\mathscr{A}_{F}\right)=\left\{E\left(f \mid \mathscr{A}_{F}\right): f \in S_{F}^{1}\right\}$ as in the above proof $(1)$. Hence $E[F]=E[G]$ holds if $F(\omega), G(\omega) \in \mathscr{K}_{c}(\mathfrak{X})$ a.s. in (3). These are not true generally. In fact, let $\Omega=\Omega_{0} \cup\left\{\omega_{1}\right\}$, where $\mu\left(\Omega_{0}\right)=\mu\left(\left\{\omega_{1}\right\}\right)=1 / 2$ and $\Omega_{0}$ is nonatomic. Define $F$, $G \in \mathscr{M}[\Omega ; \mathscr{K}(\mathbf{R})]$ by $F(\omega)=G\left(\omega_{1}\right)=\{0,1\}, F\left(\omega_{1}\right)=G(\omega)=\{0\}, \omega \in \Omega_{0}$. Then $F$ and $G$ are identically distributed, but $E[F]=[0,1 / 2]$ and $E\left[F, \mathscr{A}_{F}\right]=E[G]=$ $\{0,1 / 2\}$.

We now establish the infinite dimensional version of multivalued strong law of large numbers given in [3, Theorem 3.2].

THEOREM 3.2. If $\left\{F_{n}\right\}$ is a sequence of i.i.d. random variables in $\mathscr{M}[\Omega ; \mathscr{K}(\mathfrak{X})]$ and $S_{F_{1}}^{1} \neq \varnothing$, then

$$
\frac{1}{n} \mathrm{cl} \sum_{i=1}^{n} F_{i}(\omega) \rightarrow \overline{\operatorname{co}} E\left[F_{1}\right] \text { a.s. }
$$


Proof. Let $X=\overline{\operatorname{co}} E\left[F_{1}\right]$ and $G_{n}(\omega)=n^{-1} \mathrm{cl} \sum_{i=1}^{n} F_{i}(\omega), \omega \in \Omega, n \geqslant 1$. For any $x \in X$ and $\varepsilon>0$, by Lemma 3.1(1) and (3) we can choose $f_{j} \in S_{F_{j}}^{1}\left(\mathscr{A}_{F_{j}}\right), 1 \leqslant j \leqslant m$, such that $\left\|m^{-1} \sum_{j=1}^{m} E\left(f_{j}\right)-x\right\|<\varepsilon$. By Lemma 3.1(2), there exists a sequence $\left\{f_{n}\right\}$ of $f_{n} \in S_{F_{n}}^{1}\left(\mathscr{A}_{F_{n}}\right)$ such that $f_{(k-1) m+j}, k \geqslant 1$, are identically distributed for each $j=1, \ldots, m$. Let $x_{j}=E\left(f_{j}\right), 1 \leqslant j \leqslant m$. If $n=(k-1) m+l$, where $1 \leqslant l \leqslant m$, then

$$
\begin{aligned}
\| \frac{1}{n} \sum_{i=1}^{n} f_{i}(\omega)- & \frac{1}{m} \sum_{j=1}^{m} x_{j} \| \\
= & \left\|\frac{1}{n} \sum_{j=1}^{m} \sum_{i=1}^{k} f_{(i-1) m+j}(\omega)-\frac{1}{n} \sum_{j=l+1}^{m} f_{(k-1) m+j}(\omega)-\frac{1}{m} \sum_{j=1}^{m} x_{j}\right\| \\
\leqslant & \frac{k}{n} \sum_{j=1}^{m}\left\|\frac{1}{k} \sum_{i=1}^{k} f_{(i-1) m+j}(\omega)-x_{j}\right\|+\frac{k}{n} \sum_{j=1}^{m} \frac{1}{k}\left\|f_{(k-1) m+j}(\omega)\right\| \\
& +\left(\frac{k}{n}-\frac{1}{m}\right)\left\|\sum_{j=1}^{m} x_{j}\right\| .
\end{aligned}
$$

For $1 \leqslant j \leqslant m$, since $\left\{f_{(k-1) m+j}: k \geqslant 1\right\}$ is a sequence of i.i.d. random variables in $L^{1}(\Omega ; \mathfrak{X})$, it follows that

$$
\left\|\frac{1}{k} \sum_{i=1}^{k} f_{(i-1) m+j}(\omega)-x_{j}\right\| \rightarrow 0 \text { a.s. as } k \rightarrow \infty
$$

and hence $k^{-1}\left\|f_{(k-1) m+j}(\omega)\right\| \rightarrow 0$ a.s. as $k \rightarrow \infty$. Therefore

$$
\left\|\frac{1}{n} \sum_{i=1}^{n} f_{i}(\omega)-\frac{1}{m} \sum_{j=1}^{m} x_{j}\right\| \rightarrow 0 \quad \text { a.s. as } n \rightarrow \infty \text {. }
$$

Since $n^{-1} \sum_{i=1}^{n} f_{i}(\omega) \in G_{n}(\omega)$ a.s., we have $m^{-1} \sum_{j=1}^{m} x_{j} \in \mathrm{s}-\liminf G_{n}(\omega)$ a.s. Thus $X \subset \mathrm{s}-\lim \inf G_{n}(\omega)$ a.s.

Next let $\left\{x_{j}\right\}$ be a sequence dense in $\mathfrak{X} \backslash X$. By the separation theorem, there exists a sequence $\left\{x_{j}^{*}\right\}$ in $\mathfrak{X}^{*}$ with $\left\|x_{j}^{*}\right\|=1$ such that

$$
\left\langle x_{j}, x_{j}^{*}\right\rangle-d\left(x_{j}, X\right) \geqslant s\left(X, x_{j}^{*}\right), \quad j \geqslant 1 .
$$

Then $x \in X$ if and only if $\left\langle x, x_{j}^{*}\right\rangle \leqslant s\left(X, x_{j}^{*}\right)$ for all $j \geqslant 1$. Because the function $X \mapsto s\left(X, x_{j}^{*}\right)$ of $\mathscr{K}(\mathfrak{X})$ into $(-\infty, \infty]$ is $\mathscr{B}_{\mathscr{K}(\mathfrak{X})}$-measurable and

$$
E\left(s\left(F_{1}(\cdot), x_{j}^{*}\right)\right)=s\left(X, x_{j}^{*}\right)<\infty, \quad j \geqslant 1,
$$

$\left\{s\left(F_{n}(\cdot), x_{j}^{*}\right): n \geqslant 1\right\}$ is a sequence of i.i.d. random variables in $L^{1}$ for each $j \geqslant 1$. So there exists a $\mu$-null set $N \in \mathscr{A}$ such that, for every $\omega \in \Omega \backslash N$ and $j \geqslant 1$,

$$
s\left(G_{n}(\omega), x_{j}^{*}\right)=\frac{1}{n} \sum_{i=1}^{n} s\left(F_{i}(\omega), x_{j}^{*}\right) \rightarrow s\left(X, x_{j}^{*}\right) \quad \text { as } n \rightarrow \infty .
$$

If $x \in \mathrm{w}-\lim \sup G_{n}(\omega)$ for $\omega \in \Omega \backslash N$, then $x_{k} \stackrel{\mathrm{w}}{\rightarrow} x$ for some $x_{k} \in G_{n_{k}}(\omega)$ and hence

$$
\left\langle x, x_{j}^{*}\right\rangle=\lim _{k}\left\langle x_{k}, x_{j}^{*}\right\rangle \leqslant \lim _{k} s\left(G_{n_{k}}(\omega), x_{j}^{*}\right)=s\left(X, x_{j}^{*}\right), \quad j \geqslant 1,
$$

which implies $x \in X$. Thus w-lim $\sup G_{n}(\omega) \subset X$ a.s. Q.E.D. 
REMARK. C. Hess (Loi forte des grands nombres pour des ensembles aléatoires non bornés à valeurs dans un espace de Banach séparable, C. R. Acad. Sci. Paris Sér. I 300 (1985), 177-180) independently proved Theorem 3.2 for the pairwise independent, identically distributed case.

We finally obtain another type of multivalued strong laws of large numbers. Concerning strong laws for only independent Banach space-valued random variables, the main results are as follows (cf. $[6,19,20,34]): ~ \mathfrak{X}$ is $B$-convex (resp. of type $p$, where $1 \leqslant p \leqslant 2$ ) if and only if $\left\|n^{-1} \sum_{i=1}^{n} f_{i}(\omega)\right\| \rightarrow 0$ a.s. for any sequence $\left\{f_{n}\right\}$ of independent random variables in $L^{2}(\Omega ; \mathfrak{X})$ (resp. $L^{p}(\Omega ; \mathfrak{X})$ ) with $E\left(f_{n}\right)=0$ and $\sup E\left(\left\|f_{n}\right\|^{2}\right)<\infty$ (resp. $\left.\sum_{n=1}^{\infty} n^{-p} E\left(\left\|f_{n}\right\|^{p}\right)<\infty\right)$. Note that $\mathfrak{X}$ is $B$-convex if and only if $\mathfrak{X}$ is of type $p$ for some $p>1$. We refer to $[30,34]$ for $B$-convexity and types of Banach spaces.

THEOREM 3.3. Suppose that $\mathfrak{X}$ is of type $p$, where $1<p \leqslant 2$. If $\left\{F_{n}\right\}$ is a sequence of independent random variables in $\mathscr{M}[\Omega ; \mathscr{K}(\mathfrak{X})]$ such that $\sum_{n=1}^{\infty} n^{-p} E\left(\left\|F_{n}(\cdot)\right\|^{p}\right)<\infty$ and if there exists an $X \in \mathscr{K}(\mathfrak{X})$ such that

$$
\begin{gathered}
X \subset \mathrm{s}-\lim \inf \mathrm{cl} E\left[F_{n}, \mathscr{A}_{F_{n}}\right], \\
\lim \sup s\left(\operatorname{cl} E\left[F_{n}\right], x^{*}\right) \leqslant s\left(X, x^{*}\right), \quad x^{*} \in \mathfrak{X}^{*},
\end{gathered}
$$

then

$$
\frac{1}{n} \mathrm{cl} \sum_{i=1}^{n} F_{i}(\omega) \rightarrow \overline{\operatorname{co}} X \text { a.s. }
$$

Proof. Let $G(\omega)=n^{-1} \operatorname{cl} \sum_{i=1}^{n} F_{i}(\omega)$. For any $x \in \overline{\operatorname{co}} X$ and $\varepsilon>0$, select $x_{1}, \ldots, x_{m}$ $\in X$ such that $\left\|m^{-1} \sum_{j=1}^{m} x_{j}-x\right\|<\varepsilon$. By condition (3.1), there exists a sequence $\left\{f_{n}\right\}$ of $f_{n} \in S_{F_{n}}^{1}\left(\mathscr{A}_{F_{n}}\right)$ such that $\left\|E\left(f_{(k-1) m+j}\right)-x_{j}\right\| \rightarrow 0$ as $k \rightarrow \infty$ for each $j=1, \ldots, m$. Let $y_{n}=E\left(f_{n}\right), n \geqslant 1$. If $n=(k-1) m+l$, where $1 \leqslant l \leqslant m$, then

$$
\begin{aligned}
\left\|\frac{1}{n} \sum_{i=1}^{n} f_{i}(\omega)-\frac{1}{m} \sum_{j=1}^{m} x_{j}\right\| \leqslant & \left\|\frac{1}{n} \sum_{i=1}^{n}\left(f_{i}(\omega)-y_{i}\right)\right\|+\left\|\frac{1}{n} \sum_{i=1}^{n} y_{i}-\frac{1}{m} \sum_{j=1}^{m} x_{j}\right\| \\
\leqslant & \left\|\frac{1}{n} \sum_{i=1}^{n}\left(f_{i}(\omega)-y_{i}\right)\right\|+\frac{k}{n} \sum_{j=1}^{m} \frac{1}{k} \sum_{i=1}^{k}\left\|y_{(i-1) m+j}-x_{j}\right\| \\
& +\frac{1}{n} \sum_{j=1}^{m}\left\|y_{(k-1) m+j}\right\|+\left(\frac{k}{n}-\frac{1}{m}\right)\left\|\sum_{j=1}^{m} x_{j}\right\| .
\end{aligned}
$$

Since $\left\{f_{n}\right\}$ is a sequence of independent random variables in $L^{p}(\Omega ; \mathfrak{X})$ with $\sum_{n=1}^{\infty} n^{-p} E\left(\left\|f_{n}\right\|^{p}\right)<\infty$, it follows that $\left\|n^{-1} \sum_{i=1}^{n}\left(f_{i}(\omega)-y_{i}\right)\right\| \rightarrow 0$ a.s. Therefore

$$
\left\|\frac{1}{n} \sum_{i=1}^{n} f_{i}(\omega)-\frac{1}{m} \sum_{j=1}^{m} x_{j}\right\| \rightarrow 0 \text { a.s. as } n \rightarrow \infty,
$$

so that $m^{-1} \sum_{j=1}^{m} x_{j} \in \mathrm{s}-\lim \inf G_{n}(\omega)$ a.s. Thus $\overline{\operatorname{co}} X \subset \mathrm{s}-\lim \inf G_{n}(\omega)$ a.s.

Let $\left\{x_{j}^{*}\right\}$ be as in the proof of Theorem 3.2 taken for $\overline{\operatorname{co}} X$. Then $\left\{s\left(F_{n}(\cdot), x_{j}^{*}\right)\right.$ : $n \geqslant 1\}$ is a sequence of independent random variables in $L^{p}$ with $\sum_{n=1}^{\infty} n^{-p} E\left(\left|s\left(F_{n}(\cdot), x_{j}^{*}\right)\right|^{p}\right)<\infty$. Further, by (3.1), (3.2) and Lemma 3.1(1),

$$
E\left(s\left(F_{n}(\cdot), x_{j}^{*}\right)\right)=s\left(\operatorname{cl} E\left[F_{n}\right], x_{j}^{*}\right) \rightarrow s\left(X, x_{j}^{*}\right) \quad \text { as } n \rightarrow \infty .
$$


Hence, for each $j \geqslant 1$, we have $s\left(G_{n}(\omega), x_{j}^{*}\right) \rightarrow s\left(X, x_{j}^{*}\right)$ a.s. as $n \rightarrow \infty$. Thus w-lim $\sup G_{n}(\omega) \subset \overline{\operatorname{co}} X$ a.s. follows as in the proof of Theorem 3.2. Q.E.D.

Corollary 3.4. Suppose that $\mathfrak{X}$ is B-convex. If $\left\{F_{n}\right\}$ is a sequence of independent random variables in $\mathscr{M}[\Omega ; \mathscr{K}(\mathfrak{X})]$ such that $\sup E\left(\left\|F_{n}(\cdot)\right\|^{2}\right)<\infty$ and if there exists an $X \in \mathscr{K}(\mathfrak{X})$ satisfying (3.1) and (3.2) in Theorem 3.3, then

$$
\frac{1}{n} \operatorname{cl} \sum_{i=1}^{n} F_{i}(\omega) \rightarrow \overline{\operatorname{co}} X \quad \text { a.s. }
$$

REMARK. Condition (3.1) in Theorem 3.3 seems somewhat unpleasant. However cl $E\left[F_{n}, \mathscr{A}_{F_{n}}\right]$ may be replaced by cl $E\left[F_{n}\right]$ if $F_{n}(\omega) \in \mathscr{K}_{c}(\mathfrak{X})$ a.s. for all $n \geqslant 1$ (see remark to Lemma 3.1). If $\mathrm{cl} E\left[F_{n}, \mathscr{A}_{F_{n}}\right] \stackrel{h}{\rightarrow} X$, then (3.1) and (3.2) are satisfied. Condition (3.2) implies

$$
\text { w-lim sup cl } E\left[F_{n}\right] \subset \overline{\operatorname{co}} X,
$$

and vice versa when $\mathfrak{X}$ is reflexive and, as in Corollary 3.4 , sup $E\left(\left\|F_{n}(\cdot)\right\|\right)<\infty$ (see Lemma 1.1(1) and (2)).

\section{REFERENCES}

1. Z. Artstein, A note on Fatou's lemma in several dimensions, J. Math. Econom. 6 (1979), 277-282.

2. Z. Artstein and J. C. Hansen, Convexification in limit laws of random sets in Banach spaces, Ann. Probab. 13 (1985), 307-309.

3. Z. Artstein and S. Hart, Law of large numbers for random sets and allocation processes, Math. Oper. Res. 6 (1981), 485-492.

4. Z. Artstein and R. A. Vitale, A strong law of large numbers for random compact sets, Ann. Probab. 3 (1975), 879-882.

5. R. J. Aumann, Integrals of set-valued functions, J. Math. Anal. Appl. 12 (1965), 1-12.

6. A. Beck, On the strong law of large numbers, Ergodic Theory (F. B. Wright, ed.), Academic Press, New York, 1963, pp. 21-53.

7. C. Castaing and M. Valadier, Convex analysis and measurable multifunctions, Lecture Notes in Math., vol. 580, Springer-Verlag, Berlin, 1977.

8. N. Cressie, A strong limit theorem for random sets, Suppl. Adv. in Appl. Probab. 10 (1978), 36-46.

9. G. Debreu, Integration of correspondences, Proc. Fifth Berkeley Sympos. on Math. Statist. and Prob., Vol. II, Part I, Univ. of California Press, 1967, pp. 351-372.

10. E. Giné, M. G. Hahn and J. Zinn, Limit theorems for random sets: An application of probability in Banach space results, Probability in Banach Spaces IV (A. Beck and K. Jacobs, eds.), Lecture Notes in Math., vol. 990, Springer-Verlag, Berlin, 1983, pp. 112-135.

11. S. Hart and E. Kohlberg, Equally distributed correspondences, J. Math. Econom. 1 (1974), 167-174.

12. C. Hess, Théorème ergodique et loi forte des grands nombres pour des ensembles aléatoires, C. R. Acad. Sci. Paris Sér. A 288 (1979), 519-522.

13. L_ Loi de probabilité des ensembles aléatoires à valeurs fermées dans un espace métrique séparable, C. R. Acad. Sci. Paris Sér. I 296 (1983), 883-886.

14. L__ Loi de probabilité et indépendance des ensembles aléatoires à valeurs fermées dans un espace de Banach, Séminaire d'Analyse Convexe, Montpellier, 1983, Exposé $n^{\circ} 7$.

15. F. Hiai, Strong laws of large numbers for multivalued random variables, Multifunctions and Integrands (G. Salinetti, ed.), Lecture Notes in Math., vol. 1091, Springer-Verlag, Berlin, 1984, pp. $160-172$.

16. F. Hiai and H. Umegaki, Integrals, conditional expectations, and martingales of multivalued functions, J. Multivariate Anal. 7 (1977), 149-182.

17. W. Hildenbrand and J.-F. Mertens, On Fatou's lemma in several dimensions, Z. Wahrsch. Verw. Gebiete 17 (1971), 151-155.

18. C. J. Himmelberg, Measurable relations, Fund. Math. 87 (1975), 53-72. 
19. J. Hoffmann-Jørgensen, Probability in Banach space, Ecole d'Eté de Probabilités de Saint-Flour VI-1976, Lecture Notes in Math., vol. 598, Springer-Verlag, Berlin, 1977, pp. 1-186.

20 , Probability and geometry of Banach spaces, Functional Analysis (D. Butković et al., eds.),

Lecture Notes in Math., vol. 948, Springer-Verlag, Berlin, 1982, pp. 164-229.

21. K. Kuratowski, Topology, Vol. I (Transl. from French), Academic Press, New York, 1966.

22. U. Mosco, Convergence of convex sets and of solutions of variational inequalities, Adv. in Math. 3 (1969), 510-585.

23. On the continuity of the Young-Fenchel transform, J. Math. Anal. Appl. 35 (1971), 518-535.

24. E. Mourier, L-random elements and $L^{*}$-random elements in Banach spaces, Proc. Third Berkeley Sympos. on Math. Statist. and Prob., Vol. II, Univ. of California Press, 1956, pp. 231-242.

25. M. L. Puri and D. A. Ralescu, Strong law of large numbers for Banach space valued random sets, Ann. Probab. 11 (1983), 222-224.

26. G. Salinetti and R. Wets, On the relations between two types of convergence for convex functions, J. Math. Anal. Appl. 60 (1977), 211-226.

27. , On the convergence of sequences of convex sets in finite dimensions, SIAM Rev. 21 (1979),

$18-33$.

28. , On the convergence of closed-valued measurable multifunctions, Trans. Amer. Math. Soc. 266 (1981), 275-289.

29. D. Schmeidler, Fatou's lemma in several dimensions, Proc. Amer. Math. Soc. 24 (1970), 300-306.

30. L. Schwarz, Geometry and probability in Banach spaces, Lecture Notes in Math., vol. 852, Springer-Verlag, Berlin, 1981.

31. M. Tsukada, Convergence of closed convex sets and $\sigma$-fields, Z. Wahrsch. Verw. Gebiete 62 (1983), 137-146.

32. , Convergence of best approximations in a smooth Banach space, J. Approx. Theory 40 (1984), 301-309.

33. M. Valadier, Sur l'espérance conditionnelle multivoque non convexe, Ann. Inst. Henri Poincaré Sect. B 16 (1980), 109-116.

34. W. A. Woyczyński, Geometry and martingales in Banach spaces-Part II: Independent increments, Probability on Banach Spaces (J. Kuelbs, ed.), Dekker, New York, 1978, pp. 267-517.

Department of Information Sciences, Science University of Tokyo, Noda City, Chiba 278, JAPAN

Current address: Research Institute of Applied Electricity, Hokkaido University, Sapporo 060, Japan 